Bei langem Liegen, besser und weit vollstăndiger beim Erwărmen, zerfallt das $\mathrm{H}_{8} \mathrm{O}_{2}$ in der ublichen Weise: $2 \mathrm{H}_{2} \mathrm{O}_{2} \rightarrow 2 \mathrm{H}_{2} \mathrm{O}+\mathrm{O}_{2}$.

Hiervon tritt grobtenteils $\mathrm{O}_{\mathfrak{z}}$ an die Nebenvalenz:

$$
2 \stackrel{\mathrm{NaBO}_{2}}{\mathrm{H}_{2} \mathrm{O}_{2}} \rightarrow \begin{gathered}
\mathrm{NaBO}_{2} \quad \mathrm{NaBO}_{8} \\
\mathrm{O}-\mathrm{O}^{\prime}
\end{gathered}
$$

während andererseits auch in geringerem Umfange die Umsetzung nach

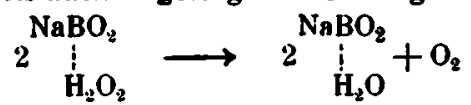

vor sich geht.

Bei Berührung mit flüssigem Wasser geht der erstere Kðrper unter stürmischer Sauerstoffentwicklung in letzteren tiber:

$$
{ }_{\mathrm{O}-\mathrm{O}}^{\mathrm{NaBO}_{2}}+2 \mathrm{HaBO}_{2} \mathrm{O} \longrightarrow 2 \stackrel{\mathrm{H}_{2} \mathrm{O}}{\mathrm{NaOO}_{2}}+\mathrm{O}_{2} \text {. }
$$

Stimnt auch die hier ingenommene Formel $\left(\mathrm{NaBO}_{2}\right)_{2} \mathrm{O}_{2}$ mit der Bruttoformel $\mathrm{NaBO}_{3}$ liberein, :o zeigt sie doch, daB die bei weitgehendem Entwăssern des Perborats entstehende Verbindung ganz andere, etwa an die des $\mathrm{K}_{2} \mathrm{O}_{2}\left(\mathrm{~K}_{2} \mathrm{O}_{2} \cdot \mathrm{O}_{2}\right)$ erinnernde, Eigenschatten hat, als man sie für einen Korper der Formel $\mathrm{NaBO}_{3}$ erwarten sollte, nach der man geneigt ist, ein Substitutionsprodukt des $\mathrm{H}_{8} \mathrm{O}_{2}$, etwa $\mathrm{NaOB}\langle\underset{0}{0}$ 7.u erwarten. Dieses mübte analog den Persulfaten oder Percarbonaten wohl eine verhältnismäßig hohe, nicht : iber eine äulberst verminderte Beständigkeit besitzen. Dabs beim Entwässern des Perborats ein Übergang von einem Additions- in ein substitutionsprodukt des $\mathrm{H}_{2} \mathrm{O}_{8}$ hochstwahrscheinlich nicht eintritt, lehrt der Umstand, daß auch beim Eintragen des bei 55" entwäisserten oder des auf 120" erhitzten Produktes in starke Jodkaliumlosung nicht mehr als Spuren freien Jods neben $\mathrm{O}_{2}$ auftreten.

Fur die Auffassung des Natriumperborales als eines Substitutionsproduktes des $\mathrm{H}_{3} \mathrm{O}_{2}$ könnte die Tatsache ins Feld geführt werden, daß es - scheinbar in Analogie mit solchen - nach K. Arndt (Z. Elektroch. 22, 63 [1916]) auch elck/rolytisch dargestellt werden kann. Dies ist aler nur möglich in einer mit Soda versetzten Boraxlosung. Vergleiclit man aber den zeitlichen Verlanf der Stromausbeute an aktivem Sauerstoff einmal in einer mit Borax rersetzten und dann in einer auf et wa gleiche $\mathrm{OH}^{\prime}$-Konzentration durch $\mathrm{NaHCO}_{3}$ abgestimmten Sodalösung, so zeigt sich vollstăndiges Zusammenfallen beider Kurven. In lelzterem Falle entsteht P'ercarbonat, welches nach $\mathrm{Na}_{2} \mathrm{C}_{2} \mathrm{O}_{6}+2 \mathrm{H}_{2} \mathrm{O}$ $2 \mathrm{NiHCO}+\mathrm{H}_{2} \mathrm{O}_{2}$ in L,osung Wasserstof fsuperoxyd liefert. Dadur'b ist bewiesen, dals dis: elektrolytische Perboratbildung kein primärer Anodenvorgang ist, sondern nur sekundär durch Vernittelung des primär entstindenen Percarbonats vor sich geht und darum auf die Mitwirkung der Soda angewiesen ist.

Sebr bemerlienswert erscheint schließlich die Tatsache, daß durch Zutrilt des $\mathrm{H}_{2} \mathrm{O}$, an das Metatorat das entstehende Perborat sich als das Salz einer Sïure von erbeblich größurer Stärke erweist, als es die Borsiiure ist. Obgleich in einer $\mathrm{NaHCO}_{3}-\mathrm{I}$. ösung durch $\mathrm{Na}_{2} \mathrm{~B}_{4} \mathrm{O}_{7}$ die $\mathrm{OH}^{\prime}$ Konzentration el was zunimmt, kaun min. wenn zugleich neutralisiertes H.O., zugefügt wird, auch dinn, wenn noclı keine Perboratkristallisation die Gileichgewichte stort, durch einen Luftstrom sehr weitgehend die Kohlensăure gemäß einer Umsetzung natch:

$$
\begin{aligned}
& \mathrm{Nil}_{2} \mathrm{~B}_{4} \mathrm{O}-2 \mathrm{NaHCO}_{::} \text {，'4 } \mathrm{Na} 33 \mathrm{O}_{2}+2 \mathrm{H}_{2} \mathrm{CO}_{3} \\
& +\mathrm{NaBO}_{2}+4 \mathrm{H}_{2} \mathrm{O}_{2} \text {, ' }+\mathrm{NaBO}_{2} \cdot \mathrm{H}_{2} \mathrm{O}_{2}
\end{aligned}
$$

austroiben. Die nihere Verfolgung des hieraus sich ergebenden Stärkezuwachses der Borstiture ist in Angriff genommen. Man kann sich vielleicht vorstellen, daß3 die Elektroaffinitat des Boratoms durch An-

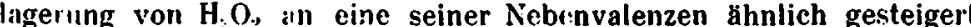
wird wie die des Wisserstoffatoms durch Anlagerung von $\mathrm{NH}_{3}$ im Ammonium.

Ijie praktisrhe liedeutung des Natriumperborates liegt in seine geringen Looslichkeit. Diese crlaubt 7. 13. aus einer $0,5 \%: 0 \mathrm{H}_{2} \mathrm{O}_{2}$ enthaltenden Lösung bei (0" etwa zwei Mriltel dieser Verbindung in ciestalt des lirislillis:erten und sehr hal baren Perborats zur Abscheidung zu brinıren. Dildurch kann unter inderem nach dem eleganten Verfaluren ron Henkel \& Co. (D.R.P. 302 7\%5) die von M. Traube zuerst beobachtete kalinodische Reduzierbarkeit des Sauerstoffs 2.11 Wasserstoffsuperoxyd ter:hnisch in einf:ichster Weise verwertet werden. Dieses Verfihren beruht, wie dic Untersuchun sen des Vortrayenden gelelirt habell, darauf, dit3 an anlilgamierten Kulfer- oder Sitherdrahtnetz-



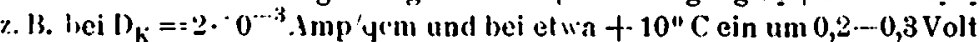
niedrigeres Kathudenpotential beanspruct $\mathrm{I}$ ins der Vorgang $\mathrm{H}_{2} \mathrm{O}_{2}+2 \mathrm{H}$

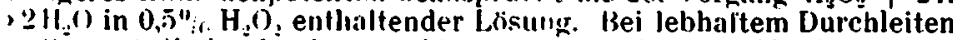
con Sauerstoff durb eine verdünnte, a chuach saure Nitriumsulfatlissung kann man daher unter dell genia inten Bedingungen H.O, von solelier Konzentration mit s(0" "Stromall sbeute erhalten. [A. 139.]
Tetralin als Reaktionsretardator bei der Herstellung von Faktis.

\author{
Von Dr. Rudolf Ditmar, Graz.
}

(Bingeg. 18.5. 1981.)

Mehr denn je muß man heute im deutschen Wirtschaftsleben darauf bedacht sein, die Einfuhr von Rohstoffen aus dem Auslande zu verringern und alle ausländischen Produkte durch Erzeugnisse des eigenen Landes nach Tunlichkeit zu ersetzen. Von diesem Gesichtspunkte ausgebend habe ich versucht, das Tetralin in der Faktisindustrie an Stelle der sonst ublichen Verdunnungsmittel als Reaktionsverzogerer bei der Einwirkung von Schwefel und Schwefelchlorür auf die zur Herstellung von Faktis verwendeten Öle in Anwendung $\mathbf{z u}$ bringen.

Die Herstellung von Faktis kann ich als bekannt voraussetzen; ich habe darüber eingehend berichtet in der Zeitschrift für angewandte Chemie 1914, I. Bd., S. 537-543 im Artikel: "Schwefelcblorüte und geschwefelte Oole" und in der Gummi-Zeitung, Berlin, 28. Jahrg., Nr. 26 ff., S. 61 im Artikel: "Einiges aber Faktis".

"Tetralin" ist ein ungiltiges Hydrierungsprodukt des Naphthalins, es siedet bei $205-210^{\circ}$, hat ein spez. Gew. von 0,975 , einen Flammpunkt von $79^{\circ}$ und einen Gefrierpunkt von unter - $20^{\circ}$. Ihm verwandt ist das "Tetralin extra". Beide Stoffe werden von der Tetralingesellschaft m. b. H. in Berlin hergestellt und finden in der Lackindustrie und Firnisindustrie als Terpentinolersatz, ferner bei der Herstellung von Schuhcreme und Bohnermassen, im graphischen Gewerbe und in der Gummiindustrie ') Verwendung. Im Kriege wurde das Tetralin als Betriebsınittel für die Unlerseeboote benutzt, da die Mineralöldestillate knapp wurden.

Seine Eigenschaft, sich mit trocknenden ölen in jedem Verhiiltnis zu mischen, legte den Gedanken nahe, die in der Faktisindustrie verwendeten Öle, wie Rübol, Leinôl, Mohnöl, Baumwollsamenôl, Rizinusöl usw. vor der Behandlung mit den Faktisbildnern Schwefel und Schwefelchlorür in Tetralin oder in Tetralin extra zu lösen. Durch die Verdünnung deröle mit den durch katalytische Hydrogenisation erhaltenen flussigen Hydronaphthalinen, wie Tetrahydronaphthalin, Dekahydronaphthalin und den $z$ wischen diesen beiden Hydrierungsprodukten liegenden Produkten (vgl. D. R. P. Nr. 320807, Kl. 22 h, Gr. 1) tritt eine we sentliche Verzogerung und bedeutende Herabminderung der stürmischen Reaktion bei der Einwirkung der Faktis. bildner ein. Vor allem wird dadurch die starke Erhitzung des sich bildenden Faktis vermieden und damit einer Verbrennung vorgebeugt. Ich unterlasse es mit Absicht, in die Rezeptbücher der Faktisfabriken einzugreifen und die Mischungsverhältnisse der verschiedenen Öle mit den Hydrierungsprodukten des Naphthalins anzugeben. Es hängt dies begreiflicherweise nicht bloß von den einzelnen Ölsorten und den angewandten Schwefel. und Schwefelchlorïrmengen, sondern auch von der Qualititit der Ölsorten ab. Von großem Einfluß ist es auch, ob das ôl im oxydierten Zustande oder als nichtoxydiertes öl zur Anwendung gebracht wurde und ob noch andere Substanzen, wie Parraffin und Mineralole, zugesetzt wurden.

Auf jeden Fall läbt sich durch Verwendung von Hydro. naphthalinen als ölverdünnungmittel Mineralol sparen, und das ist filr das deutsche Wirtschaftsleben von aus schlaggebender Bedeutung.

[A. 96.]

\section{Bemerkungen zur Abhandlung von Marcusson und Picard: „Zusammensetzung von Hoch- und Tieftemperaturteeren".}

\author{
Von An. Grex, Aussig. \\ (Kingeg. 14. i. 1!r?1)
}

In ihrer interessanten Mitteilung über „\%usammensetzung von Hoch- und Tieftemperalurteeren" beschäftigen sich Marcusson und Picard') anch mit der Frage der Bildung von Erdöl aus Fettsituren auf dem Wege iber die Ketone. Sic erwaihnen dibei eine einschligigige Untersuchung von mir und $\mathrm{Wirth}^{*}$ ), von der sic aber verade nur das fïr die Erdôhypothese gan Unwesentliche wiedergeben, und die sie denientsprechend auch unrichtig glossieren. Sie sclureiben nämlic:h: "In Jalıre 1920 luben Grïn und Wirth erneut aluf die Moglichlieit einer Ketonbildung bei der Erdölentstehung hingewiesen. Sie \%eisten, dats beim Destillieren von slearinsaurem Satron im Vakuum nehen Kohlenwasserstoffen ein festes Keton, das Pentatriakontamon, entsteht, welches sich bei gewöhnlicher Destillation unter Bildung von Kolılenwasserstoffen zersetzt. Nun verlinft aber die Fidolibildung wohl kaum im Valsuum, sondern eher unter Öberdruck. Wemgegenïlicr bedeuten die bei Untersuchung der beiden Braunkohlenteere erhaltenen Frgebnisse insofern eiren fortschritt, als hier \%un ersten Male bei

1) "Auto-Technik", Verlag Klasing \& Co., Berlin, Nr. ;, X. Jalurg.

1) \%eitschr. f. angew. Chemie 34, 201 [1921]

2) "Öber d’’n l'rsprung des Erdöls. Bildung aus Trejen Fetıäurev oder Seifen; Bildung aus animalischen Koblenwasserstoffen." B. 53, 1301 [1920]. 
einem Prozeß, welcher der Erdölbildung ïhnelt, erhebliche Mengen von Ketonen nachgewiesen sind."

Diese Ausführungen müssen den Eindruck erwecken, daß ich und Wirth aus der Darstellung des Ketons der Stearinsäure durch Destillation ihres Natronsalzes in Vakuum irgendwelchen Schluß auf die Erdölbildung gezogen hätten. Gerade das Gegenteil ist der Fall! Wir zeigten vielmehr, daß die Limwandlung von Fettsäuren in Erdöl auf den Wege über die Ketone erfolgt sein kann, daß aber die Ketone durchaus nicht aus fettsauren Salzen, sondern direkt aus den freien Fettsïuren entstanden sein dürften. Wir geben ausdrücklich an, daß die Annahme der intermediären Bildung von Kalksalzen oder anderen Seifen ganz überflüssig ist, weil $\ldots . .$. auch freie Fettsäuren leicht in Ketone ïbergeführt werden können, nicht nur Dämpfe flüchtiger Siluren durch gewisse Katalysatoren, sondern auch freie Fettsïuren vom höchsten Molekulargewicht, im flüssigen Zustande, durch die Einwirkung katalytischer Substanzen - es genügt sogar die bloße Berührung mit einer sehr heißen Gefäßwand“. Als Beleg für die letzte Angabe wurde das D. R. P. 296, 677 (Firma Georg Schicht A.-G. und Ad. Grün) zitiert; in der Patentbeschreibung wird, nebenbei bemerkt, auch angegeben, daß die Reaktion selbst bei relativ hohem Überdruck noch glatt von statten geht. Was die zweite l'hase, die Umwandlung der Ketone in Kohlenwasserstoffe betrifft, so haben wir ebenfalls ausdrücklich bemerkt, da13 sie unter Druck ebenso gut oder vielmehr, wie zu erwarten, besser verläuft wie im Vakuum. Wir schrieben .... bei der DestilLation unter Atmosphärendruck tritt fast vollständige Zersetzung - Krackung - ein, wobei sich mehr ungesättigte als gesättigte Kohlenwasserstoffe bilden" und belegten diese Angabe im Versuchsteil auch zahlenmäßig. Übrigens sagen Marcusson und Picard ja selbst, dab sich nacth unserer Angabe das Pentatriakontanon ,bei gew öhnlicher Destillation unter Bildung von Kohlenwasserstoffen zersetzt". Es ist mir deshalb unverstïndlich, wie Marcusson ironisch schreiben kann, ,nun verlïuft aber die Erdölbildung wohl kaum im Vaku um, sondern eher unter Überdruck ", und wie er gerade dara us den Schluß ziehen kann, daß die Ergebnisse seiner Untersuchung insofern einen Fortschritt bedeuten, "als hier zum ersten Male bei einem Prozeß, weleher der Erdölbildung ähnelt, erhebliche Mengen von Ketonen narhgewiesen sind". Irh habe nicht nur die gewöhnlichen Fettsïuren, wie Stearinsïure usw. durch bloBes Erhitzen, ohne Destillation, auch unter Drurk (also durch einen Prozeß, welcher der Erdölbildung doch wenigstens ebensosehr ähnelt wie die Teerdestillation) ketonisiert, sondern speziell auch mit Ulbrich ${ }^{3}$ ) die Montansïure auf diesem Wege in ihr Keton verwandelt, ferner diese von uns als Montanon bezeichnete Verbindung zuerst im Montanwachs aus den Schwelkohlen autgefunden. Wir haben das Montanon sowohl isoliert, als auch mittels einer für diesen Zweck ausgearbeiteten Methode indirekt quantitativ bestimmt: 3ehandeln des Wachses mit Natrium und Amylalkohol, darauf quantitative Bestimmung der neugebildeten Hydroxylgruppen im Redultionsprodukt mittels der Hydroxylzahl. Es ist daher im Grunde genommer nicht überraschend, daß Marcusson und Picard mit Hilfe dieser Methode auch in den Braunkohlenteeren unverindert geblicbenes Montanon nachweisen konnten. Jedenfalls freut es mich, daß ein so erfahrener Analytiker wie Marcusson unsere Methode akzeptiert und mit Erfolg angewendet hat.

\section{Kieselgur zum Zurückhalten von Niederschlägen.}

\section{Von Dr. Zinkeisen, Hamburg.}

$$
\text { (Einger. 10. 6. 1921.) }
$$

Als Ergänzungr zu den in dieser Zeitschrift $(34$, S. 242, 1921) von Bruhns gemachten Mitteilungen möchte ich noch darauf hinweisen, daß Kieselgur nicht nur mechanisch feste Schwimmstoffe zurückhält, sondern vorteilhaft auch zum Klarfiltrieren von wässerigen Flüssigkeiten verwendet werden kann, die durch ölige Substanzen getrübt sind. Ich habe diese Eirfahrung bei alkoholischen Auszügen aus Zitronenschalen gemacht, aus welchen die Terpene durch Zusatz von Wasser ausgeschieden wurden. Die Terpent: sammeln sich zum größten Teil auf der Flüssigkeit, ein Teil bleibt aber suspendiert und geht beim Filtrieren ohne Kieselgur mit durch das Filter. Schüttelt man aber eine kleine Mense vorher mit Kieselgur und gibt sie alsdann auf das Filter, so können ganz erhebliche weitere Mengen klar filtriert werden. Die zurïckhaltende Kraft der Kieselgur (Adsorption?) geht sogar so weit, dal zuletzt eine dünne Ölschicht auf der Flüssigkeit im Filter schwimmt, ohne daß das Öl durch das Filter geht. [A. 133.]

\section{Personal- und Hochschulnachrichten.}

Die diesjährige Handels-Hochschul-Konferenz fand an den letzten drei Tagen der Pfingstferien in der Berliner Handels-Hochschule statt. Den wichtigsten Gegenstand der Beratungen bildete die Frage der Angliederung der Handels-Hochschulen an Universitäten oder Technische Hochsichulen. Die Rektorenkonferenz ist nach eingehenden Beratungen zu den Beschiuß gekommen, daß gegen jede Art der

3) Zur Kenutnis: des Montanwachses, Chem. Umschau 23, 57 [1916]; Chem. Umschau 24, $43 \mid 1917]$.
Verschmelzung einer Handels-Hochschule mit anderen Hochschulen große Bedenken bestehen, wenn nicht die Erfüllung der bisherigen Aufgaben der Handels-Hochschule in vollem Umfange gewährleistet bleibt. Gegen Arbeitsgemeinschaften mit anderen Hochschulen wurden keine Bedenken erhoben. Von weiteren Beratungsgegenständen sind zu erwähnen die Bedingungen für die Zulassung zum Handels-Hochschul-Studium und die Regelung der Gebühren. Die Hochschulvertreter haben beschlossen, einstweilen keine Erhöhung der Gebühren vorzuschlagen. Zum nächsten Vorort der deutschen Handels-Hochschulen wurde an Stelle des bisherigen Vororts Berlin die HandelsHochschule Mannheim gewählt.

Ehrungen: Geheimrat Prof. Dr. C. Duisberg wurde zum Dr. phil. nat. h. c. der Naturwissenschaftl. - mathematischen Fakultät der Ruperto Carola Universität Heidelberg ernannt. - Die Technische Hochschule Karlsruhe ernannte den Generaldirektor der Pumpen- und Armaturenfabrik Klein, Schanzlin u. Becker, A.-G., Frankenthal (Pfalz), Kommerzienrat J. Kl ein, zum Ehrendoktor.

Es wurden berufen: Der o. Dozent am Polytechnikum zu Cöthen, Dipl.-Ing. E. Diepschlag, zum o. Professor für Eisenhüttenkunde an der Technischen Hochschule in Breslau als Nachfolger des Professors Oberhoffer; Prof. Dr. E. Müller, Abteilungsvorsteher am chemischen Laboratorium der Universität Heidelberg, in gleicher Eigenschaft an die Universität Köln.

Gestorben ist: Prof. Dr. Th. Diehl, stellvertretender Vorsitzender des Vereins deutscher Chemiker, am 2. 7. in Darmstadt im 67. Lebensjahre.

\section{Bücherbesprechungen.}

Oetling, Dr. C., Schmiede und Schmiedetechnik. Ein Handbuch für Betriebsleiter Schmiedemeister und Studierende. Band I: Die Brennstoffe, die Erwärmungsvorrichtungen für feste Brennstoffe und zugehörige Meßinstrumente, handbediente Vorrichtungen, Werkzeuge, Maschinen, ausschließlich Druckluftmaschinen, sowie zugehörige Prüfvorrichtungen, Transport- und Bedienungsmittel. Mit 606 Textabbildungen. Druck und Verlag von R. Oldenbourg, München und Berlin 1920.

Das umfangreiche Werk von 608 Seiten ist entstanden aus einer Preisarbeit des Verfassers auf Grund eines Preisausschreibens des Vereins deutscher Maschineningenieure über Schmiedetechnik aus dem Jahre 1911. Die Arbeit ist alsdann vom Verfasser ausgebaut worden. Fast alle wesentlichen Schmiedehilfsmittel sind systematisch zusammengefaßt und nicht nur beschreibend erwähnt, sondern auch zahlenmäßig, d. h. in ihrer Wirtschaftlichkeit dargelegt worden. Die Öfen, Maschinen und Apparate sind im Wirklichkeitsbild, zum Teil in Strichzeichnung wiedergegeben. Die Abmessungen sind meistens tabellarisch zusammengestellt, ebenso die wirtschaftlichen Ergebnisse. Die Wärmebilanzen der Öfen sind klar herausgeholt und die theoretischen Grundlagen ebenfalls dargelegt worden. Besonders hervorzuheben sind auch wertvolle Kurventafeln, die eine gute Übersicht über alle Faktoren geben. Daß das Werk dazu beiträgt, den Wert guter Wärmeanlagen für die Großschmiede klar vor Augen zu führen und dadurch die Grundlage zu geben für weitere Verbesserungen, insbesondere durch Anwendung der nötigen Meßverfahren zur Betriebskontrolle, sei lobend erwähnt.

Auch die verschiedenen mechanischen Hämmer sind gut dargestellt, nur wären hier mehr Strichzeichnungen am Platze gewesen. leider nur stiefmütterlich behandelt. Die nächste Auflage könnte diesem Mangel abhelfen. Etwa das letzte Drittel des Buches dient den Pressen und den Maschinen zum Trennen, also den Schneid- und Lochmaschinen, für welche dasselbe gilt wie für die Hämmer. Die Schweißmaschinen sind in einem kurzen Kapitel von 6 Seiten 7.1 kurz behandelt. Sehr wertvoll ist das Kapitel über Meßinstrumente und Meßmethoden für Arbeitsmaschinen, welches jedoch ebenfalls: noch weiter ausgebaut werden kann. Die Transport- und Bedienungsmittel, die am Schlusse des Werkes zusammengestellt sind, könnte man vielleicht ganz entbehren und auf die entsprechende umfängliche Literatur verweisen. Will der Verfasser aber für die nächste Auflage diesen Abschnitt aufrechterhalten, so muß auch er weiter ausgebaut werden. Die überaus fleißige Arbeit des Verfassers verdient jedoch Einführung in die weitesten Kreise der interessierten Fachwelt, zumal die vollkommene Schmiedetechnik in den meisten Werken nicht zu ihrem Rechte kommt.

Freund. [BB. 141.]

Das Ganze der Fabrik-Buchhaltung nach doppeltem und amerikanischem System für höhere Textilschulen, höhere Handelsschulen, verwandte Lehranstalten und für das Geschäftsleben von Prof. Ed. Feuerstein. Dritte Auflage, besorgt von Handelsschulleiter Dr. O. Hertel. Verlag der modernen kaufmännischen Bibliothek G.m.b. H., Leipzig-Reudnit

Preis geb. $\mathrm{M} \mathrm{10}-\cdots+20 \%$ Teuerungszuschlag.

Durch methodische Behandlung und Auswahl des Stoffes nach den Bedürfnissen der Praxis zeichnet sich das Lehrbuch vor vielen dieser Art aus. Das Buch ist deshalb auch zum Selbststudium allen Fachgenossen zu empfehlen, die sich mit dem Wesen der doppelten Buchbaltung vertraut machen wollen. 